\title{
ORGANISATION OF KNOWLEDGE FROM TRACES OF HUMAN LEARNING
}

\author{
Baba Mbaye \\ EFFET B company, France, Laboratory of ELLIADD, University of Franche-comtè, France
}

\begin{abstract}
Faced with the diversity and the large number of learning resources available on e-learning platforms, learners often face problems of inadequacy between the training path to choose and the skills sought.

Indeed, many learning platforms with recommendation exist, but these do not often include information and different characteristics of learners. This problem can be mitigated by the semantisation of the content and the organization of the knowledge of the different actors involved on the learning platform.

In this paper, we make a modeling of the knowledge representation (thesauri and concept maps) of learning resources, based on information from learner's written records on a learning platform and knowledge modeling from expert interactions on resource and learner assessments.

These models make it possible to represent knowledge from written tracesthese written traces are metadata resulting from the interactions made on a learning platform by the learners and the experts (for example the expert in charge of the content of the trainings, the expert in charge of the evaluation of the trainings carried out).
\end{abstract}

\section{KEYWORDS}

E-Learning, Semantic Network, Knowledge Representation, Semantic Web, Written Records

\section{INTRODUCTION}

Knowledge is the activity by which man seeks to understand reality. The term "representation" will be examined here in the context of the theory of knowledge. In fact, knowledge representation is a set of tools and technologies. In our research, this set is used to represent on the one hand and on the other hand to organize human knowledge for the purpose of using it in an automated way and to share it (Luca Stefanutti et al, 2017).

The organization of knowledge consists of making a representation of a set of knowledge in a formalized and schematic way. It is an essential link in the process of managing knowledge and skills in an organization. Knowledge modeling is an activity at the center of many knowledge management occupations by positioning knowledge modeling at their center (Rappin, Baptiste, 2014).

\section{CONTRIBUTION}

The purpose of this research is to refine the content of the learning platform (CLP) and to facilitate the management of online CLP by opting for the relevance, accessibility of knowledge useful to learners. This knowledge is refined and adapted according to the learning pathways of the learners. The management of the CLP is facilitated by the reproduction of the actions of the experts on the learning platform in a automat. 


\section{CONCEPTS, PROCESSING AND MODELING}

\subsection{Information and Knowledge}

It is important at first to present the difference between the concepts of information and knowledge. Information is the set of all data external to people, communicated orally or mediated in documents. Whereas knowledge is the result of any mental construct internationalized by an individual from information he obtains.

The interpretation of information by a person consists in transforming these into internal knowledge in his memory, which can also be called "learning". Knowledge representation is the reverse process by which a person produces information usable by other people, using a representation system, which can also be called "knowledge extraction" (Sanetake Nagayoshi et al, 2017).

The processing of information and knowledge, the acquisition, the construction of work processes require a system of representation external to our memory. The history of mankind is rich in such systems, starting with oral, written or pictorial languages that allow objects and ideas and ideas to be represented by words or symbols and to establish relations between these objects and these ideas.In the fields of mathematics and science, the geometric representations and Cartesian diagrams that we study in primary or secondary school are other examples of commonly usedrepresentation systems(Mostafa Al-Emran et al, 2018).

In the early sixties, the evolution of cognitive sciences and computer science led to the creation of knowledge representation systems such as concept maps, semantic networks, schemas or semantic frameworks, entity-relationship models, information flow models, object-oriented models, etc (Antonio Lieto et al, 2018).

The purpose of these systems is to propose a formal language, often graphic, to make a representation of the knowledge that "hide" in the information, what is called in English "knowledge level". For example, two texts, one in French and one in English, translation from one another, will be represented in the same way by a knowledge model that groups together the concepts that these texts deal with and the relationships between them. concepts. Similarly, an order describing a work process and a written text describing the same sequence of operations will be represented in a similar way in a knowledge representation system (Xing Tang et al, 2019 ). This is called representation of the meaning of a document or semantic representation.A semantic representation is an image of the mental model of knowledge derived from the peculiarities of the format chosen to present the information (Zhenyong Wu et al, 2016).

\subsection{Information Processing vs. Knowledge Processing}

Information processing consists of circulating within the organization the information of the field in which the organization operates, as well as internal and external information on the goods and services produced by the organization and its working processes. Some of this information is retained and processed to constitute the memory of the organization in the form of banks of documents or database, which used to produce goods and services.

The processing of knowledge adds to this traditional process, the interpretation, ie the transformation of information into knowledge by staff through learning and the reverse process, ie the extraction and presentation of knowledge held by some members staff in the form of information that will be integrated into the organization's memory made available to all staff. In this sense, it can be said that the treatment of knowledge is characteristic of the "learning" organization (Nopparat Thepthepa et al, 2016).

The central component of a knowledge-processing system is the knowledge base, which is a standard representation of the knowledge of the domain the organization wants to deal with and which is used to make a representation of the content of the documents as well as the data found in the memory of the organization (Meenakshi Malhotra et al, 2016). The knowledge base is used to refer in an integrated way the documents and the information it contains, then to look for the information, to locate alternative, neighboring or complementary documents, or to identify the needs of the organization in terms of knowledge. not covered or covered incompletely (Layla Unger et al 2016).

The representation of knowledge is limited to the processing of documents. It is an approach that leads to unstructured and unrelated activities of creation, import, capture, research and use of knowledge. Knowledge 
components may exist in a large type of document format or in implicit knowledge held by individuals but still expressed in the organization's memory. In terms of information retrieval, documents are syntactically referenced by a ranking engine, instead of direct referencing to a knowledge model. Thus the problem is the follow-up of associations between concepts to identify related or complementary documents.

It is an association that allows IT agents to search and find documents by their semantics, their content, rather their syntax, their form.

The documents accessible by internet are associated with each other and the representation of knowledge of this association is at the bottom of the semantic web.

The process of knowledge processing has four main steps :

- The creation or import of information in the form of text, still and moving images, graphic templates, etc.

- The semantic referencing of information, doCcuments, information is indexed and described by their attribute, by metadata, by their position in classifications, e, using ontologies and generally in the framework of a system of knowledge representation.

- Research and access to information by first identifying knowledge elements in the knowledge base, rather than data in a database. The latter being referenced according to knowledge, it will be found using metadata as the basis of the research. We can also use the knowledge model or semantic referencing ontology for browsing information, doing research, getting access to web pages, documents, people.

- The use of information in the context of work processes by segmenting, aggregating, annotating and integrating information into new information resources or documents that will in turn be semantically referenced and integrated into the process of processing knowledge.

\subsection{The Graphical Representation of the Knowledge Model}

There are several techniques for representing knowledge. Each representation technique has characteristics that allow optimal adaptation to certain realities to be modeled. The characterization of objects, the study of processes, the construction of decisional or logical system, there is a technique of representation more adapted for the modeling of the system.Trees and semantic networks, flow charts, causal diagrams, decision trees, and object-oriented models are all ways of representing knowledge that is based on a graphical approach.

- Semantic tree is used a lot in pedagogy. The latter is used to highlight components of knowledge in the form of hierarchical structure starting from a general object and classification by specialization of classes into subclasses and class into individuals. There are no links between the branches of the hierarchy. This is a technique that is often used in website design.

- Semantic Network (conceptual map or relationship entity graph): a semantic network makes it possible to relate concepts and facts by the naming of links. This representation is widely used in computer science, especially for database design, thus linking tables that groups the concepts described by their attributes. In artificial intelligence a semantic network makes it possible to establish a base of facts that makes a characterization of a specific domain.

- The flowchart is used for the purpose of modeling the procedures so far the graphs represented concepts and their relationships. In a flowchart, objects are actions or decisions, and links represent the flow of information from one action or decision to another. The notion of sequence is thus introduced in this type of representation.

\section{OUR APPROACH}

We will use semantic networks for the graphical representation of our knowledge model. The semantic network simulates our representation of memory, it is a model that shows us how knowledge could be represented in memory and how we could access this knowledge. Indeed, the memory is an immense pool of raw data containing concepts, events, and written traces resulting from interaction. All these elements form an immense inter-relational network. A semantic network is composed of nodes whose interrelations are established by labeled pointers. These nodes represent the different types of knowledge in memory. These nodes can be associated with properties that apply to the networks. 


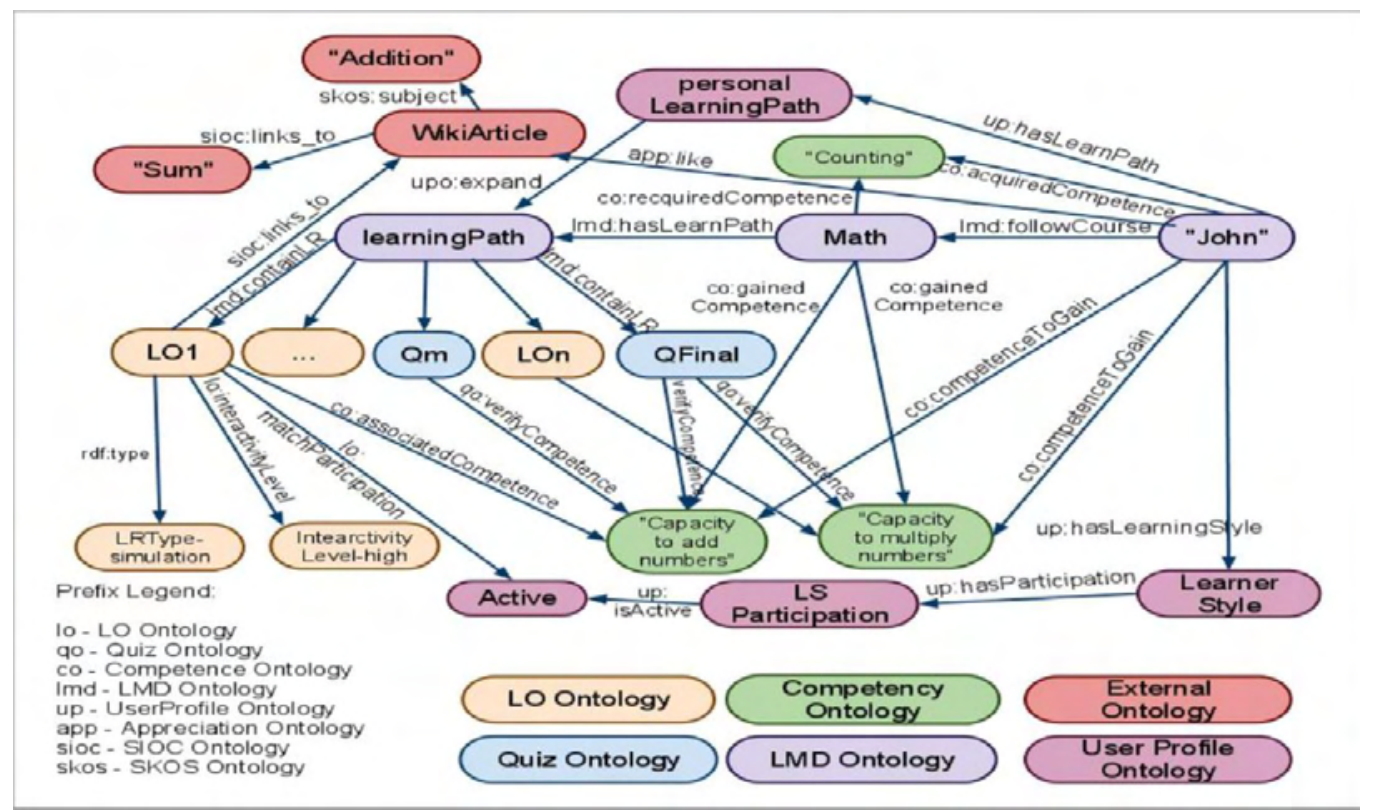

Figure 1. Example of semantic network (Ioan Szilagyi et al, 2011)

Our approach is based on reuse of the traces obtained from the interactions made by the different agents on the platform to generate a semantic network for each learner according to his profile and needs. The idea here is to set up an adaptive semantic network generator according to the needs and profile of the learner and his tutor. In our study, our traces of the various agents of the platform are stored in a LRS (learning record store) with Tin Can (TC) in Json format. The use of the traces will allow us to include the events which occurred during the execution of the trainings by the learners.

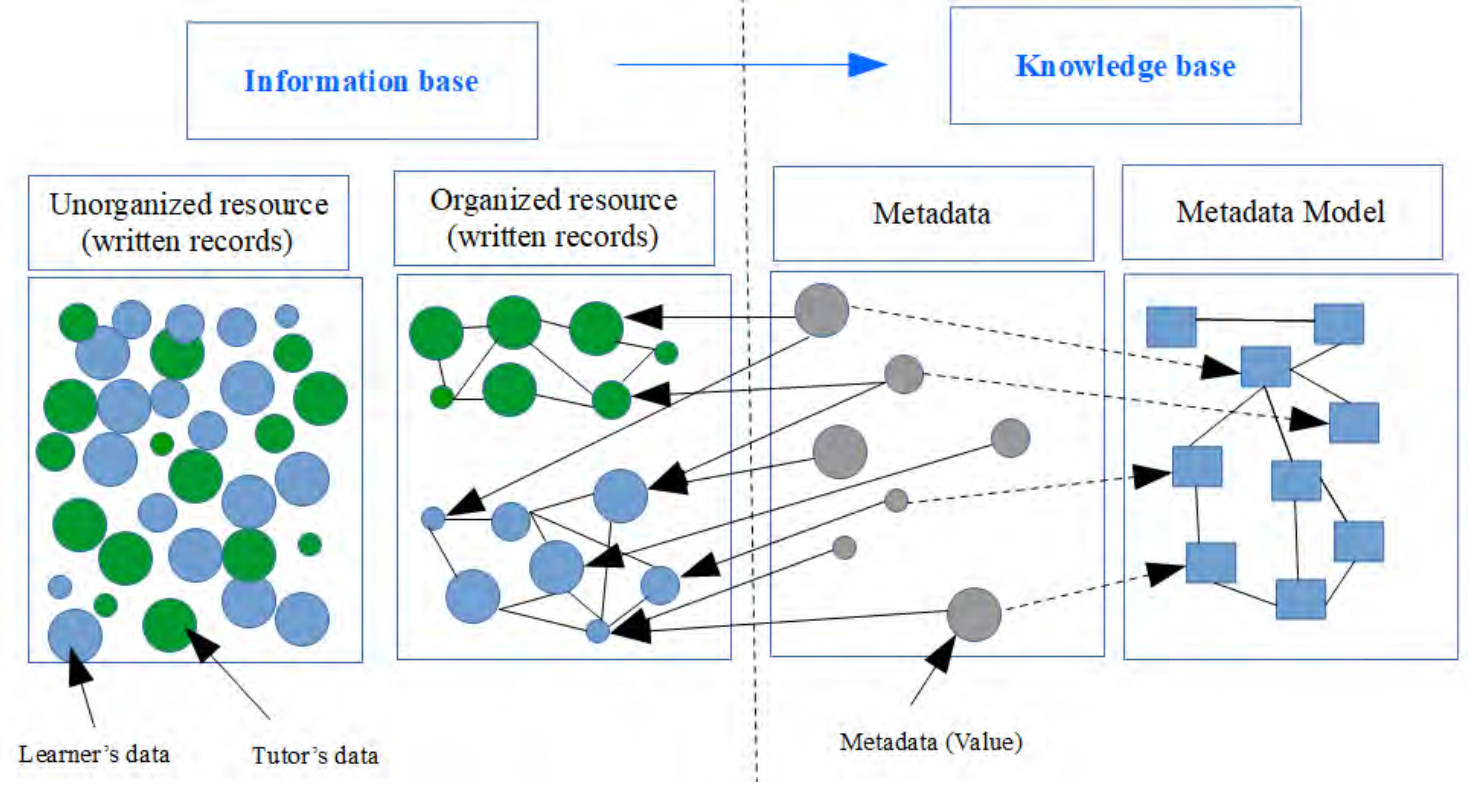

Figure 2. Written records knowledge organisation Model 


\section{CONCLUSION}

In this paper, we present a representation model of knowledge from written traces made by the agents of an e-learning platform. This study allows us to show that the content management of an e-learning platform can be done automatically, efficiently and adapt to the profile of each learner.

\section{REFERENCES}

Antonio Lieto, Christian Lebiere, Alessandro Oltramari, The knowledge level in cognitive architectures: Current limitations and possible develoments, Cognitive Systems Research, Volume 48,2018, Pages 39-55,ISSN 1389-0417

Johanna Gast, Katherine Gundolf, Rainer Harms, Elvin Matos Collado, Knowledge management and coopetition: How do cooperating competitors balance the needs to share and protect their knowledge?,Industrial Marketing Management, 2019,

Luca Stefanutti, Debora de Chiusole, On the assessment of learning in competence based knowledge space theory, Journal of Mathematical Psychology, Volume 80, 2017, Pages 22 32, ISSN 0022-2496, https://doi.org/10.1016/j.jmp.2017.08.003.

Layla Unger, Anna V. Fisher, Rebecca Nugent, Samuel L. Ventura, Christopher J. MacLellan, Developmental changes in semantic knowledge organization, Journal of Experimental Child Psychology, Volume 146, 2016, Pages 202-222, ISSN 0022-0965 https://doi.org/10.1016/j.jecp.2016.01.005.

Meenakshi Malhotra, T.R. Gopalakrishnan Nair, Knowledge network model with neurocognitive processing capabilities, Cognitive Systems Research, Volume 40, 2016, Pages 186-201, ISSN 1389-0417, https://doi.org/10.1016/j.cogsys.2015.11.002.

Mostafa Al-Emran, Vitaliy Mezhuyev, Adzhar Kamaludin, Khaled Shaalan, The impact of knowledge management processes on information systems: A systematic review, International Journal of Information Management, Volume 43,2018, Pages 173-187, ISSN 0268-4012, https://doi.org/10.1016/j.ijinfomgt.2018.08.001.

Nopparat Thepthepa, Toshioa Mitsufuji, Knowledge Process and Learning Organization Development in Science Museums, Procedia Computer Science, Volume 99, 2016, Pages 157-170, ISSN 1877-0509, https://doi.org/10.1016/j.procs.2016.09.108.

Sanetake Nagayoshi, Jun Nakamura, Accelerate Information Interpretation in the Organizational Failure Learning, Procedia Computer Science, Volume 112, 2017, Pages 971-979, ISSN 1877- 0509, https://doi.org/10.1016/j.procs.2017.08.139.

Szilagyi, I., Balog-Crisan, R., Roxin, A. and Roxin, I., 2011, July. Ontologies and Knowledge Aggregation in the Active Semantic Learning System. In 2011 IEEE 11th International Conference on Advanced Learning Technologies (pp. 388-392). IEEE.

Xing Tang, Ling Chen, Jun Cui, Baogang Wei,Knowledge representation learning with entity descriptions, hierarchical types, and textual relations, Information Processing \& Maagement,Volume 56, Issue 3, 2019, Pages 809-822, ISSN 0306-4573, https://doi.org/10.1016/j.ipm.2019.01.005.

Zhenyong $\mathrm{Wu}$, Jihua Liao, Wenyan Song, Hanling Mao, Zhenfeng Huang, Xinxin Li, Hanying Mao, Semantic hypergraph-based knowledge representation architecture for complex product development, Computers in Industry, Volume 100, 2018, Pages 43-56, ISSN 0166-3615, https://doi.org/10.1016/j.compind.2018.04.008 